\title{
Heat removing under hypersonic conditions
}

\author{
Mikhail E. Semenov ${ }^{1,2,3, a}$, Victor A. Nesterov ${ }^{4}$, Ilya E. Kuznetsov ${ }^{1}$, Andrey M. Solovyov², and Peter A. Meleshenko ${ }^{1,2, b}$ \\ 1 Zhukovsky-Gagarin Air Force Academy, Starykh Bolshevikov st. 54 “A”, 394064, Voronezh, Russia \\ 2 Digital Technologies Department, Voronezh State University, Universitetskaya sq. 1, 394006, Voronezh, Russia \\ 3 Voronezh State University of Architecture and Civil Engineering, XX-letiya Oktyabrya st. 84, 394006 Voronezh, Russia \\ 4 Moscow Aviation Institute (National Research University), Volokolamskoe shosse 4, 125993 Moscow, Russia
}

\begin{abstract}
In this paper we consider the heat transfer properties of the axially symmetric body with parabolic shape at hypersonic speeds (with a Mach number $M>5$ ). We use the numerical methods based on the implicit difference scheme (Fedorenko method) with direct method based on LU-decomposition and iterative method based on the Gauss-Seigel method. Our numerical results show that the heat removing process should be performed in accordance with the nonlinear law of heat distribution over the surface taking into account the hypersonic conditions of motion.
\end{abstract}

\section{Introduction}

Rapid development of the aviation and space technology in the second half of XX century leads to a wide and exciting interest to the problems of hypersonic flow around bodies. In order to study the processes of heat exchange and heat removing in the design of aircraft as well as to obtain the stationary aerodynamic characteristics together with the traditional theoretical and experimental methods the methods of numerical simulation are widely used. The efficient numerical algorithms provide a possibility to obtain the data on the structure of flows around streamlined surfaces due to its comparative cheapness as well as to possibility to reproduce the natural conditions that are unattainable in the laboratory .

The thermal protection of the construction is a one of the most difficult problems in the interaction of high-enthalpy gas flows with aircraft. In order to realize the thermal protection in practice the passive and active methods are used, as well as the combined method: blowing the cooling gas in high-enthalpy gas flow from the porous structural elements combined with a flow of heat at the surface due to the choice of high thermal conductivity material for the composite shell. Study of the heat transfer (and heat removing) at these conditions is performed by solving the direct and inverse problems. In present time it is especially important to use the methods for solving the inverse problems due to the lack of the information on the processes under consideration as well as due to increasing requirements for the accuracy of determining of heat transfer characteristics of the streamlined body's shell taking into account the nonlinearity, multi-dimensionality and manyparametric character of the heat and mass transfer processes.

There are many works that are dedicated to the hypersonic aerodynamic regimes. However the problems of heat transfer and heat removing at hypersonic speeds remain

\footnotetext{
a e-mail: mkl150@mail.ru

b e-mail: melechp@yandex.ru
}

not well studied. For example, an important problem is the high-temperature thermomechanical behavior of composite materials based on thermostable matrices and fillers [1$3]$. At the same time the problem of numerical modeling of heat transfer on the surface of hypersonic aircraft [4-6] is also very actual and promisingly.

\section{Heat transfer on parabolic surface}

In this paper we consider a numerical simulation of the heat exchange on the surface of the axially symmetric body with parabolic shape at hypersonic speeds, which is a part of hypersonic aircraft (Fig. 1).

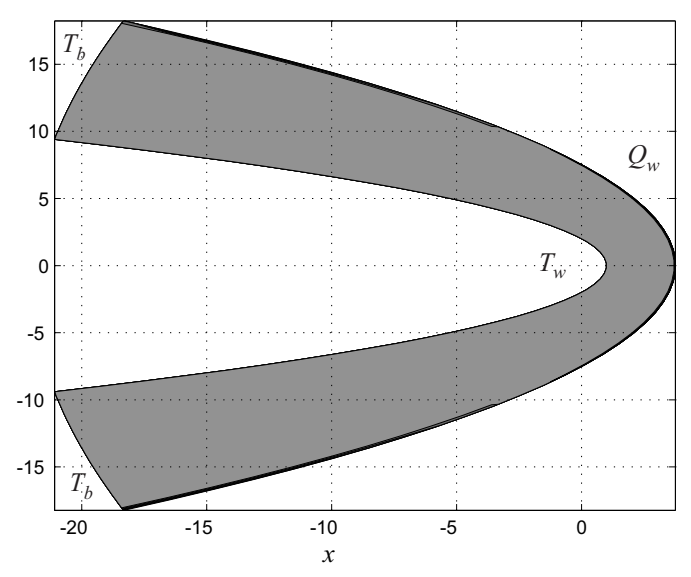

Fig. 1. Parabolic section of a body which is a part of hypersonic aircraft

In order to describe the distribution of heat on a flat surface we use the two-dimensional heat equation (equa- 
tion of heat diffusion):

$$
\frac{\partial T(x, y, t)}{\partial t}=D\left(\frac{\partial^{2} T(x, y, t)}{\partial x^{2}}+\frac{\partial^{2} T(x, y, t)}{\partial y^{2}}\right)+\varphi(x, y, t, T),
$$

where $D$ is a value which determines the rate of heat flow from the heated region to a less heated and may be a function of the coordinates and temperature. The function $\varphi(x, y, t, T$ describes the influx of heat from outside, namely the heat sources that may also depend on the spatial coordinates (localization of sources), the time and temperature.

To simplify the modeling problem we will consider the special case where the stationary source of heat works for a long time and is determined by the initial conditions, and the transition processes caused by its appearance are ended.

In this case the heat conduction equation (1) is converted to the standard Laplace equation:

$$
\frac{\partial^{2} T(x, y, t)}{\partial x^{2}}+\frac{\partial^{2} T(x, y, t)}{\partial y^{2}}=0 .
$$

Taking into account the fact that the studied surface has a parabolic shape it is more appropriate to search the solutions of the equation (2) in the parabolic coordinates (Fig. 2).

\subsection{Some notations on parabolic coordinates}

Here we would like to recall some basic properties of the parabolic coordinates. The two-dimensional parabolic co-

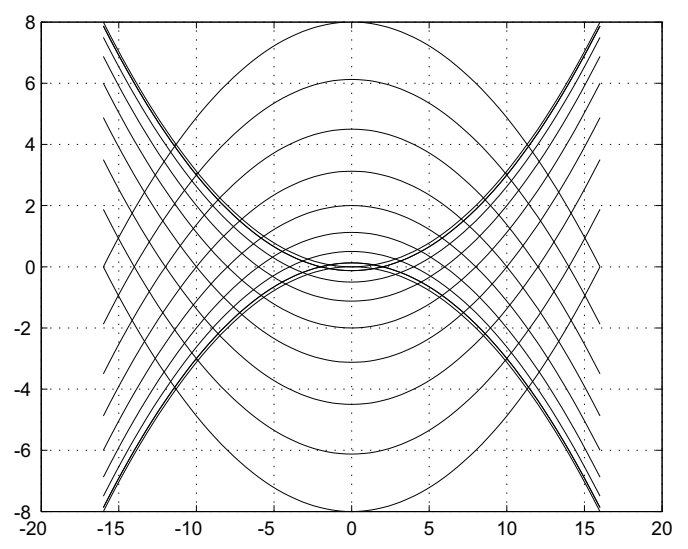

Fig. 2. Parabolic coordinates

ordinates are determined by the expression:

$$
\left\{\begin{array}{c}
x=\sigma \tau \\
y=\frac{1}{2}\left(\tau^{2}-\sigma^{2}\right)
\end{array}\right.
$$

while the surfaces of constant $\sigma$ are confocal parabolas

$$
2 y=\frac{x^{2}}{\sigma^{2}}-\sigma^{2}
$$

expanding upward (along the ray $+y$ ), and the surfaces of constant $\tau$ are confocal parabolas

$$
2 y=-\frac{x^{2}}{\tau^{2}}-\tau^{2},
$$

expanding downward (along the ray $-y$ ). Focuses of all the parabolas are located at the origin.

It is obvious that the surface shown in Fig. 1 is a flat plate in parabolic coordinates, and the solution of the temperature distribution problem becomes simpler.

Let us consider the Laplace operator $\Delta$ in parabolic coordinates. Lame coefficients for parabolic coordinates are

$$
H_{\sigma}=H_{\tau}=\sqrt{\sigma^{2}+\tau^{2}},
$$

the area element is

$$
\mathrm{d} S=\left(\sigma^{2}+\tau^{2}\right) \mathrm{d} \sigma \mathrm{d} \tau,
$$

and the action of the Laplace operator has the form:

$$
\Delta \Phi=\frac{1}{\sigma^{2}+\tau^{2}}\left(\frac{\partial^{2} \Phi}{\partial \sigma^{2}}+\frac{\partial^{2} \Phi}{\partial \tau^{2}}\right) .
$$

Thus, the Laplace equation (2) in the parabolic coordinates can be written as:

$$
\frac{\partial^{2} T(\sigma, \tau)}{\partial \sigma^{2}}+\frac{\partial^{2} T(\sigma, \tau)}{\partial \tau^{2}}=0, \sigma \in[0, G], \tau \in[0, H] .
$$

We assume that on the inner surface of the body there is a parabolic temperature distribution defined by the law $T_{w}(\sigma)$. In the field of $T_{b}$ the heat exchange with the environment is absent and the temperature on the outer surface of the body depends on a number of aerodynamic parameters and describes by the function $Q_{w}(\sigma)$. Then the boundary conditions for the equation (9) can be written as follows:

$$
\left\{\begin{array}{l}
T(\sigma, 0)=T_{w}(\sigma), \\
T(\sigma, H)=Q_{w}(\sigma), \\
\frac{\partial T}{\partial \sigma}=0 \text { at } \sigma=0, \\
\frac{\partial T}{\partial \sigma}=0 \text { at } \sigma=G .
\end{array}\right.
$$

\subsection{Heat transfer under hypersonic condition}

As was shown in [7], in the case of hypersonic speeds, the heat distribution law $Q_{w}(\sigma)$ on the surface of the axially symmetric body will be determined by the following expression:

$$
Q_{w}(\sigma)=F\left(M_{\infty}, \sigma\right)\left[\frac{1+0.096 \sqrt{\varepsilon}}{1.068}\right] \frac{1-g_{w}}{1-g_{w s}},
$$

where $\varepsilon$ is a coefficient depending on the speed of the external environment, $g_{w}$ is an enthalpy of the outer surface of the body, $g_{w s}$ is a critical enthalpy;

$$
\begin{aligned}
& F\left(M_{\infty}, \sigma\right)=2 \sigma \sin \sigma\left[\left(1-\frac{1}{\gamma_{\infty} M_{\infty}^{2}}\right) \cos ^{2} \sigma+\right. \\
& \left.+\frac{1}{\gamma_{\infty} M_{\infty}^{2}}\right] \frac{1}{\sqrt{D\left(M_{\infty}, \sigma\right)}},
\end{aligned}
$$


where $\gamma_{\infty}$ is a heating rate, $M_{\infty}$ is a Mach number, and

$$
\begin{aligned}
& D\left(M_{\infty}, \sigma\right)=\left(1-\frac{1}{\gamma_{\infty} M_{\infty}^{2}}\right)\left[\sigma^{2}-\frac{\sigma \sin 4 \sigma}{2}+\right. \\
& \left.+\frac{1-\cos 4 \sigma}{8}\right]+\frac{4}{\gamma_{\infty} M_{\infty}^{2}}\left[\sigma^{2}-\sigma \sin 2 \sigma+\frac{1-\cos 2 \sigma}{2}\right]
\end{aligned}
$$

Thus, the solution of equation (9) with the boundary conditions (10) using (11)-(13) determines the temperature distribution over the entire surface of the parabolic body which is a part of the hypersonic aircraft.

\section{Numerical simulation}

\subsection{Difference scheme}

Let us make the numerical simulation of the heat transfer on the surface of parabolic body by means of numerical solution of the equation (9). Let us approximate the equation (9) and the boundary conditions (10) on the five-point pattern of the "cross"-type. In this case we get the implicit difference scheme in the form

$$
\left\{\begin{array}{l}
\frac{T_{i+1}^{j}-2 T_{i}^{j}+T_{i-1}^{j}}{h_{i}^{2}}+\frac{T_{i}^{j+1}-2 T_{i}^{j}+T_{i}^{j-1}}{h_{j}^{2}}=0, \\
T\left(i h_{i}, 0\right)=T_{w}\left(i h_{i}\right), \\
T\left(i h_{i}, M h_{j}\right)=Q_{w}\left(i h_{i}\right), \\
\frac{T_{1}^{j}-T_{0}^{j}}{h_{i}}=0, \\
\frac{T_{N}^{j}-T_{N-1}^{j}}{h_{i}}=0, \\
i=\overline{0, N}, j=\overline{0, M},
\end{array}\right.
$$

where $h_{i}$ is a step along the $\sigma$-axis and $h_{j}$ is a step along the $\tau$-axis.

Let us prove the stability of the presented difference scheme with respect to perturbations of the boundary conditions using the spectral method [8]. We represent the solution of the system (14) in the form of harmonics

$$
T_{k}^{p}=\lambda^{p} \exp (i \alpha k) .
$$

Substitution of (15) in to the first equation of the system (14) and simplification of the obtained expression gives

$$
\frac{e^{i \alpha}+e^{-i \alpha}-2}{h_{i}^{2}}+\frac{(\lambda-1)^{2}}{\lambda h_{j}^{2}}=0 .
$$

The difference scheme is stable in the case of performing the required spectral Neumann conditions:

$$
|\lambda(\alpha)| \leqslant 1 .
$$

From equation (16) it follows that

$$
|\lambda(\alpha)|=1, \forall \alpha .
$$

Thus, the spectral Neumann condition (17) is performed and the used difference scheme is absolutely stable.

Of course the system of equations (14) can be solved using the direct methods. However, for small values of $h_{i}$ and $h_{j}$ in this case we have a problem with the conditionality matrix describing the system, and this fact leads to increasing of the calculation errors. The iterative methods are connected with the searching of algorithms and parameters to ensure the optimum convergence. The most effective and used method which takes into account the above mentioned drawbacks is a Fedorenko method [9]. This method is a combination of direct and iterative methods for solving the grid problems. The essence of the method is as follows: at the first stage the required solution is obtained by direct methods such as Gaussian elimination, Cramer or LU-decomposition on the grid with a major step. Further approximation of the solutions is carried out on a grid with a fine step and made recalculation using the iterative methods such as Gauss-Seidel method with the control of the convergence process.

\subsection{Numerical results}

We solve the system (14) using the Fedorenko method [9]. As a direct method we use the LU-decomposition. As an iterative method we use the Gauss-Seidel method.

We use the following parameters of the system. Aerodynamic characteristics are: $g_{w}=0.01, g_{w s}=0.98, \varepsilon=$ 600, $M_{\infty}>5, T_{w}(\sigma)=0$. For the direct method: $h_{i}=1$, $h_{j}=0.1, N=10, M=10$. For the iterative method: $h_{i}=0.2, h_{j}=0.02, N=50, M=50$.

The simulation results are presented in Fig. 3, 4, 5.

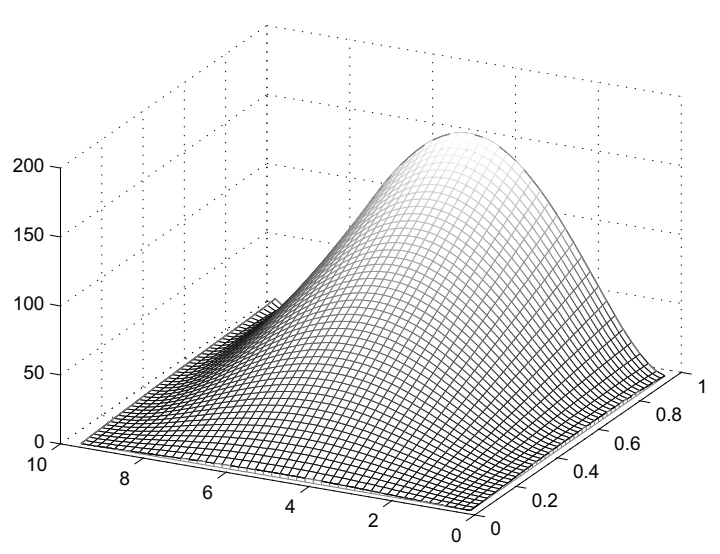

Fig. 3. Temperature distribution on the surface of parabolic body (parabolic coordinates).

As it can be seen from these figures, on the surface of the object there are regions with different degrees of heat that are nonlinearly distributed over the entire area. Therefore, if the object is a part of the hypersonic aircraft, the heat removing should be performed in accordance with the received law of distribution over the surface. 


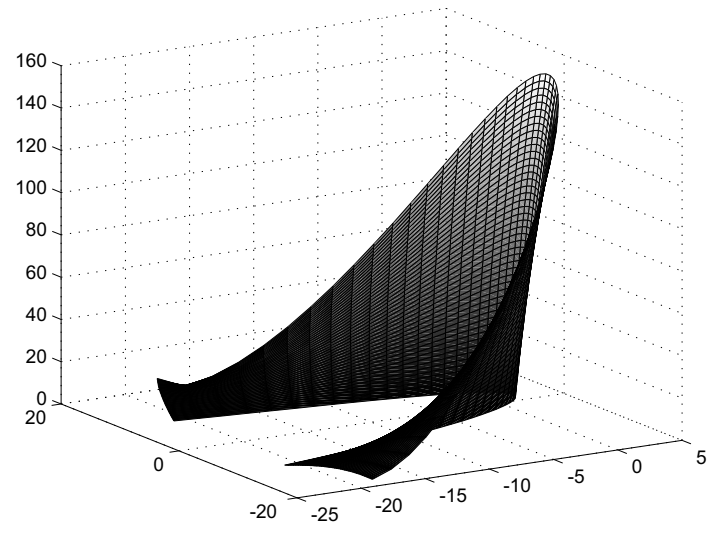

Fig. 4. Temperature distribution on the surface of parabolic body (Cartesian coordinates).

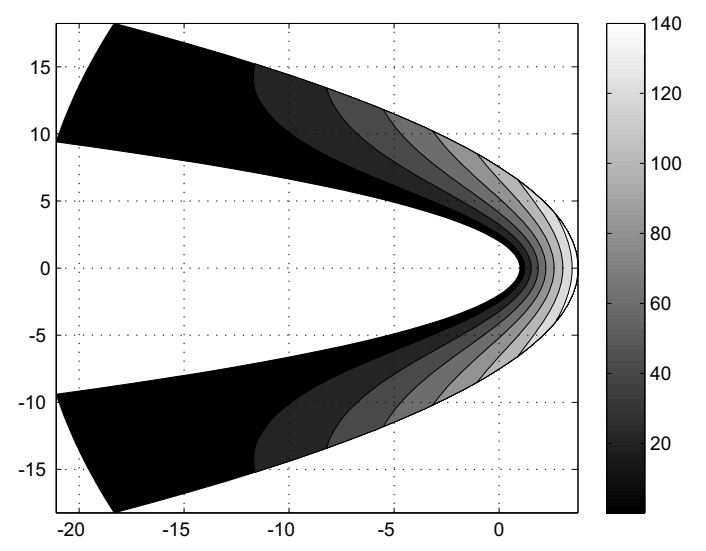

Fig. 5. Temperature distribution on the surface of parabolic body (contour plot).

\section{Conclusions}

In this paper we have considered the heat transfer properties of the axially symmetric body with parabolic shape at hypersonic speeds (with a Mach number $M>5$ ). We have used the numerical methods for solution of the Laplace equation in parabolic coordinates based on the implicit difference scheme (we use the Fedorenko method) with direct method based on LU-decomposition and iterative method based on the Gauss-Seigel method. The fact that the considered parabolic body moves with a hypersonic velocity has taken into account by the special form of the heat distribution law $Q_{w}(\sigma)$, i.e. the heat distribution of the air flow on the outer surface at hypersonic velocity. Our numerical results show that the heat removing process should be performed in accordance with the nonlinear law of heat distribution over the surface (which is solution of the heat equation) taking into account the hypersonic conditions of motion.

\section{References}

1. Y.I. Dimitrienko, Thermomechanics of Composites under High Temperatures (Springer, 1999) 350pp

2. Y.I. Dimitrienko, Int. J. Heat Mass Transf. 38, (1995) 139

3. Y.I. Dimitrienko, Int. J. Eng. Sci. 35, (1997) 15

4. V.I. Zinchenko, A.Ya. Kuzin, J. Appl. Mech. Tech. Phys. (in Russian) 43, (2002) 144

5. D.R. Ismagilov, R.V. Sidelnikov, "Almaz-Antey" Proc. (in Russian) 2, (2015) 49

6. G. Han, Z. Jiang, Int. Commun. Heat Mass Transf. 43, (2013) 46

7. W. Peng, Yu. He, X. Wang, J. Zhu, J. Han, Chin. J. Aeronautics, 28, (2015) 121

8. S.K. Godunov, V.S. Ryabenkii Difference schema (Elsevier Science Ltd, 1987) 508 pp.

9. R.P. Fedorenko, USSR Comput. Math. Math. Phys., 1, (1962) 1092 